\section{CONTINUING DEVELOPMENT}

\section{The blame game}

Sir, your recent interesting case ${ }^{1}$ is not unique but, rightly, malingering and factitious disorders should always be borne in mind. However, I would respectfully point out that such teaching has been available certainly in the UK dental schools in Bristol, Edinburgh, Glasgow and London (now KCL, QMUL, UCL) where I have worked with undergraduates and postgraduates, and is available in standard texts. ${ }^{2}$ Additionally, globally most graduates do realise it is their responsibility to pursue continuing development/education.

However, it is not only patients that are subject to such behaviour. I recall one student who moved to a hospital elsewhere after graduation, who was taken to task over radiology malpractice but who denied any teaching in that subject. The student failed to bargain that we kept records not only of the display of radiology teaching boards in public areas for a year; but also teaching attendances in the topic and results of their mandatory final examination question on the topic. Of course many are now within the 'blame culture'.

C. Scully, London

1. Gayathri H, Madhan B. Case report: Malingering and factitious disorders. Br Dent J 2015; 218: 610. Published online: 12 June 2015.

2. Scully C. Scully's Medical problems in dentistry. 7th ed. Elsevier, 2014.

DOI: 10.1038/sj.bdj.2015.603

\section{IN PRACTICE}

\section{Alveolar nerve injuries}

Sir, we routinely use supraperiosteal infiltration anaesthesia (SIA) in posterior mandibular dental implant procedures. SIA not only establishes adequate anaesthesia for the whole procedure but it has also been suggested as a safer technique than

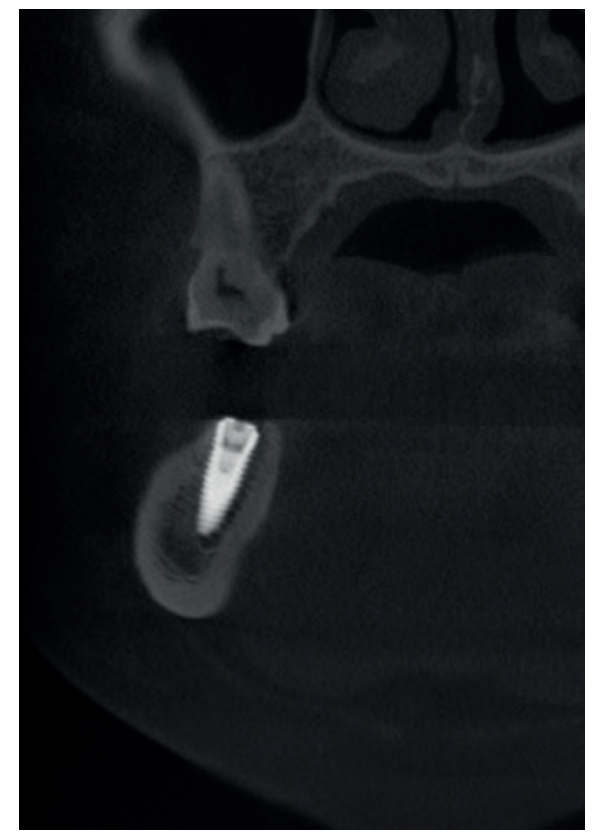

Fig. 1 The implant is touching the canal

inferior alveolar nerve (IAN) block because it supposedly allows the patient to warn us if we get too close to the IAN. ${ }^{1}$

Recently, we planned to place one implant to the right mandibular first molar region of a 42-year-old patient. After clinical examination, we took a panoramic radiograph and calculated the distance between the top of the crestal bone and IAN. For this purpose, we used specific computer software designed to used together with the panoramic device. This combination has always given very accurate measurements. Since the bone volume also seemed good, we did not need more advanced radiological techniques, such as a computerised tomography (CT).

Under SIA, we placed the implant in the usual way. During the incision, flap elevation, drilling and insertion of the implant the patient did not demonstrate any sign of pain or discomfort. After completing the procedure, we took a second panoramic radiograph in order to check the position of the implant. In this postoperative view, we noticed that the apex of the implant was in contact with the inferior alveolar canal. Since the patient was completely comfortable during the surgery, we thought that the image could be a superimposition of the implant with the IAN. We discharged the patient and told her to immediately inform us if she would feel any numbness or other discomfort in her lower lip.

Two days later, the patient contacted us and told that a numbness had started in her lower lip. We invited her and obtained a cone-beam CT. The implant was 'touching' the canal (Fig. 1). Under SIA, again, the implant was removed and a shorter implant inserted into the socket. Healing was uneventful. After three weeks, numbness was completely resolved. We did not prescribe any medication for nerve damage, such as vitamin $B$.

We think that the implant or drill just 'touched' the canal and local intraosseous oedema caused an increasing pressure on the IAN, which consequently resulted in paraesthesia. Otherwise, if the inferior alveolar canal was severely damaged, such a rapid recovery could not be seen.

We conclude that SIA could be used in dental implant procedures in mandibular molar region instead of IAN block. However, this is not a sufficient measure for preventing IAN injuries. The clinician should not rely on SIA and should give full attention to actual bone height.

K. Isik and A. Esen, Turkey

1. Heller A A, Shankland W E. 2nd Alternative to the inferior alveolar nerve block anesthesia when placing mandibular dental implants posterior to the mental foramen. J Oral Implanto/2001; 27: 127-133.

DOI: 10.1038/sj.bdj.2015.604 